\title{
Viscosity Analysis of Lubricating Oil Through the Solution of Exponential Fractional Differential Equations
}

\author{
Endang Rusyaman ${ }^{1, *}$, Kankan Parmikanti ${ }^{1}$, Diah Chaerani ${ }^{1}$, \\ Khoirunnisa Rohadatul Aisy Muslihin ${ }^{2}$
}

${ }^{1}$ Department of Mathematics, FMIPA, Universitas Padjadjaran, Indonesia

${ }^{2}$ Universitas Padjadjaran, Indonesia

\begin{abstract}
Received September 24, 2021; Revised December 15, 2021; Accepted December 23, 2021
Cite This Paper in the following Citation Styles

(a): [1] Endang Rusyaman, Kankan Parmikanti, Diah Chaerani, "Viscosity Analysis of Lubricating Oil Through the Solution of Exponential Fractional Differential Equations," Mathematics and Statistics, Vol.10, No.1, pp. 134-139, 2022. DOI: 10.13189/ms.2022.100110

(b): Endang Rusyaman, Kankan Parmikanti, Diah Chaerani, (2022). Viscosity Analysis of Lubricating Oil Through the Solution of Exponential Fractional Differential Equations. Mathematics and Statistics, 10(1), 134-139. DOI: 10.13189/ms.2022.100110
\end{abstract}

Copyright $@ 2022$ by authors, all rights reserved. Authors agree that this article remains permanently open access under the terms of the Creative Commons Attribution License 4.0 International License

\begin{abstract}
Lubricating oil is still a primary need for people dealing with machines. The important thing of lubricating oil is viscosity which is closely related to surface tension. Fluid viscosity states the measure of friction in the fluid, while surface tension is the tendency of the fluid to stretch due to attractive forces between the molecules (cohesion). We want to know how and to what extent the relationship between viscosity and surface tension of the lubricating oil is. This paper will discuss the analysis of a model in the form of an exponential fractional differential equation that states the relationship between surface tension and viscosity of lubricating oil. The Modified Homotopy Perturbation Method (MHPM) will be used to determine the solution of the fractional differential equation. This study indicates a relationship between viscosity and surface tension in the form of fractional differential equation in which the existence and uniqueness of the solution are guaranteed. From the analysis of the solution function both analytically and geometrically supported by empirical data, it can be concluded that there is a strong exponential relationship between viscosity and surface tension in lubricating oil.
\end{abstract}

Keywords Exponential, Fractional Differential Equation, Surface Tension, Viscosity

\section{Introduction}

The development of fractional calculus, particularly on derivatives and fractional differential equations in the last 20 years has increased rapidly. Many researchers are interested in developing applications of fractional differential equations in finance, system dynamics, fluid mechanics, and others. Likewise, in terms of finding a solution, in the hope of accelerating iteration, accelerating the convergence of the completion function, or increasing the accuracy of the solution, many new methods have been developed, at least modifying the old method. This paper discusses one application of fractional differential equations in fluid mechanics, especially regarding the relationship between viscosity and surface tension of lubricating oil.

Viscosity is a measure of the thickness of a fluid which states the measure of the friction in the fluid. The more viscous a fluid is, the greater the friction in the fluid, making it harder for the fluid to flow and the harder it is for objects to move in a viscous fluid. In liquids, viscosity occurs due to the cohesion force, while in gases, it is due to collisions between molecules. The measure of the viscosity of a fluid is called the coefficient of viscosity $\eta$, whose units are $N s / \mathrm{m}^{2}$ or Pascal second. If an object moves with velocity $v$ in a fluid with a viscosity coefficient $\eta$, it implies that the object will have a frictional force of $F=k \eta v$, where $k$ is a constant that depends on the geometric shape of the object.

On the other hand, the surface tension of a fluid is a characteristic of the fluid's tendency to stretch, such that its surface seems to be covered by a membrane due to the attractive forces between its molecules (cohesion). Surface tension is the force on the surface per unit length, or $\gamma=\frac{F}{d}$ where $\gamma$ is the surface tension in units of $N / m, F$ is a force with unit $N$, and $d$ is a surface length with unit $m$.

Many researchers are interested in knowing about and de- 
termining the relationship between viscosity and the surface tension of a liquid, both empirically and theoretically. The existence of this relationship is questioned by Art Gatenby [1], although in simple terms, the relationship between the two was initiated by Pelofsky [2] who introduced the empirical relationship shaped exponential function

$$
\gamma=A e^{-} \frac{B}{\eta}
$$

where $\gamma$ is the surface tension, $\eta$ is viscosity, and $A, B$ are constants.

Since then, various formula variations expressing the relationship between surface tension and viscosity have continued to develop. For example, Schonhorn [3] expanded the Pelofsky formula by expressing the tension relationship as a combination of two types of viscosities, i.e., vapor viscosity and liquid viscosity. Likewise, Li et al. [4] made an empirical correlation, Ghatee et al. [5] through measurement of Ammonium Formate, and in the second paper, [6] which states that the relationship between viscosity and surface tension depends on temperature, especially for ionic liquids.

Several researchers found a relationship between viscosity and surface tension in fluids, including Ahmari and Amiri [7], Zheng et al. [8] who introduced the relationship in the form of a normal logarithmic function, and Queimada et al, [9] on pure and mixed n-alkanes. Queimada et al. [10] also predicts the relationship between viscosity and surface tension of the fuel, and Rusyaman et al. [11] on lubricating oil.

All formulas or models regarding viscosity and/or surface tension presented above are truly ordinary relationships, even if there are derivatives, then the relationship is non-fractional. The relationships in the form of models using fractional derivatives include Mahmood et al. [12] on the exact solution of the fractional derivative model of a non-Newtonian fluid, and Jamil et al. [13] about translational flow with fractional derivatives in fluids. Moreover, Servadei and Valdinoci, and Asgari [14, 15] who each studied of weak solutions of the fractional Laplace equation with viscosity sense, and numerical solution for solving a system of Fractional Integro-Differential. The study of viscosity in rheology, fluids that change shape due to pressure and tension, did not go unnoticed by researchers, can be seen in $[16,17]$ which discusses the fractional model of creep, relaxation, and viscosity problem in Rheology.

Viscoelasticity, a combination of viscosity and elasticity, is fascinating to study in the development of viscosity. Viscoelasticity relates to materials that show viscous (for liquid materials) and elastic (for solid materials) characteristics when deformed, such as in polymeric materials like thick gel or toluene solutions of rubber.

Started by Hayat et al. and Khan et al. [18, 19] discussing Viscoelastic fluids using Fractional Maxwell Methods. Furthermore, Feral et al. [20] focused on imitating the material CF-11 network and gelatin and compared the fractionalorder and integer-order models to characterize their behavior under harmonic mechanical loads. In addition, as can be found in [21, 22, 23, 24], new researchers have emerged who write works on viscoelasticity. Michal et al. [25] provided a method to determine viscosity at Liquid Mixtures, while
Maryam Aleem [26] discussed the analysis of a mathematical model of a fractional viscous fluid through a vertical rectangular channel. As for the issue of the existence and singularity of fractional differential equations, there have been many discussions, including Ortiz J.M and Zhang S [27, 28].

Interestingly, in the last three years, there have been publications related to Meta-analysis on the effect of wettability on the coefficient of friction on viscosity problems by Michael J. Schertzer and Patricia Iglesias [29]. Similarly, the Fourier transform for the evolutionary equation by Mahmoud M. ElBorai et al. [30] and the development of the Fourier series into a fractional Fourier series by Ahmed Bouchenak et al. [31].

Thus, of the many papers that have been used as references, none has discussed the relationship between viscosity and special surface tension in lubricating oil.

\section{Basic Theory}

In this section, we present some basic theories that support the main problem, including fractional derivatives, fractional differential equations, and existence and unique theorem.

\subsection{Fractional Derivative}

There are some versions of the definition for fractional derivatives, two of which are given below.

Definition 1. Let $\alpha$ be a real number and $n-1 \leq \alpha<n$ with $n$ is a natural number. $\alpha$-ordered fractional derivative of $f(t)$ according to Riemann-Liouville is given by

$D^{\alpha} f(t)=\frac{1}{\Gamma(n-\alpha)} \frac{d^{n}}{d t^{n}} \int_{0}^{t} \frac{f(s)}{(t-s)^{-n+\alpha+1}} d s, \alpha>0, t>0$.

Definition 2. Let $\alpha$ be a real number and $n-1 \leq \alpha<n$ with $n$ is a natural number. $\alpha$-order fractional derivative of $f(t)$ according to Caputo is given by

$$
D^{\alpha} f(t)=\frac{1}{\Gamma(n-\alpha)} \int_{0}^{t} \frac{f^{(n)}(s)}{(t-s)^{-n+\alpha+1}} d s .
$$

From the two versions of the definition above, it can be concluded that the fractional derivative of $f(t)=t^{p}$ with order $\alpha$ is

$$
D^{\alpha} x^{p}=\frac{\Gamma(p+1)}{\Gamma(p-\alpha+1)} x^{p-\alpha} .
$$

The difference between the two definitions is that when $f(t)$ is a constant function, $D^{\alpha} f(t)$ is zero according to Caputo, but not zero according to Riemann-Liouville.

\subsection{Fractional Differential Equation}

One of the general forms of fractional differential equation with order $\alpha \in \mathcal{Q}$ is

$$
D^{\alpha} u(t)+L(u(t))+N(u(t))=A(t)
$$

with $t>0$ and $m-1<\alpha \leq m$, where $D^{\alpha}$ is operator of fractional derivative with order $\alpha, L$ is linear operator, $N$ is nonlinear operator, and $A(t)$ is a function of $t$. 
The more specific form of (2) is

$$
y^{(\alpha)}+c_{1} y^{2}+c_{2} y=A(t)
$$

where $c_{1}, c_{2}$ is real constants, $y$ is solution function, and $A(t)$ is a function of $t$.

\subsection{Existence and Uniqueness}

The following theorems guarantee that the solution of a fractional differential equation exists and is unique.

Theorem 1. A contractive operator that maps a Metric Space to itself has only one fixed point [27].

Proof. Let $f:(X, \rho) \rightarrow(X, \rho)$ be a contractive function, that is, there exist $c ; 0<c<1$ such that $|f(x)-f(y)| \leq$ $c|x-y|$ for every $x, y \in X$. So for any $x_{0} \in X$, we have the iterative sequence $x_{0}, x_{1}=f\left(x_{0}\right), x_{2}=f\left(x_{1}\right), x_{3}=$ $f\left(x_{2}\right), \ldots, x_{n}=f\left(x_{n-1}\right)$ converge to $a$, such that the following estimation holds

$$
\rho\left(x_{n}, a\right) \leq \frac{c^{n}}{1-c} \rho\left(x_{0}, f\left(x_{0}\right)\right) .
$$

Hence $\rho(f(a), a)=0$ or $a$ is fixed point.

Theorem 2. Let $0<\alpha<1, I=[0, h] \subset \mathbf{R}$ and $J=\left[y_{0}-\right.$ $\left.k, y_{0}+k\right] \subset \mathbf{R}$. Let $f: I \times J \rightarrow \mathbf{R}$ be a bounded continuous function, i.e., there is $M>0$ so that $|f(x, y)| \leq M$ for all $(x, y) \in I \times J$. Assume that $f$ satisfies Lipschitz property. If $L k<M$ then there is a single $y \in C\left(\left[0, h^{*}\right]\right)$, where

$$
h^{*}=\min \left\{h,\left(\frac{k \Gamma(\alpha+1)}{M}\right)^{1 / \alpha}\right\} \text {, }
$$

which applies to fractional differential equation problem

$$
D^{\alpha} y(x)=f(x, y(x)), y(0)=y_{0}[27] .
$$

Theorem 3. Fractional Differential Equation problem

$$
D^{\alpha} u+a u=q(t), t \in(0, T] \text { with } u(0)=u_{0}
$$

where $a$ is constant and $q \in C([0, T])$ has a unique solution [28].

\section{Main Result}

We take 25 samples of lubricating oil from various brands and types, then we measure the viscosity and surface tension in the laboratory. The result is shown in Table 1.

Through the scatter plot of the data, it is predicted that one of the suitable curve forms is the exponential function. Therefore, it can be assumed that the form of the fractional differential equation is also an exponential fractional differential equation.
Table 1. Viscocity and Surface-Tension on Room Temperature $20^{\circ} \mathrm{C}$.

\begin{tabular}{|c|c|c|}
\hline No & Viscosity & Surface-Tension \\
\hline 1 & 296 & 19.22 \\
2 & 406 & 19.00 \\
3 & 342 & 19.25 \\
4 & 260 & 18.98 \\
5 & 339 & 19.12 \\
6 & 193 & 18.68 \\
7 & 257 & 18.94 \\
8 & 367 & 19.13 \\
9 & 50 & 18.14 \\
10 & 277 & 18.68 \\
11 & 189 & 18.58 \\
12 & 363 & 19.03 \\
13 & 246 & 18.68 \\
14 & 225 & 18.77 \\
15 & 184 & 18.80 \\
16 & 336 & 19.29 \\
17 & 350 & 19.25 \\
18 & 230 & 19.01 \\
19 & 183 & 18.74 \\
20 & 207 & 18.95 \\
21 & 234 & 18.88 \\
22 & 361 & 19.19 \\
23 & 229 & 18.97 \\
24 & 205 & 18.58 \\
25 & 269 & 18.74 \\
\hline
\end{tabular}

\subsection{Prediction of the Exponential Fractional Differential Equation Model}

Based on the preliminary results above, the prediction of the exponential fractional differential equation model is

$$
\gamma^{(\alpha)}+\gamma=b e^{h \eta} \text { or } \gamma^{(\alpha)}=b e^{h \eta}-\gamma
$$

with $0<\alpha<1$ and boundary condition $\gamma(0)=\gamma_{0}$.

To solve the exponential fractional differential equation in (4) several methods can be used. In this paper, we will use is the Modified Homotopy Perturbation Method (MHPM).

The steps taken are as follows. (4) is a special form of (2) where $L(\gamma(\eta))$ and $N(\gamma(\eta))=0$, and $A\left((\eta)=b e^{h \eta}\right)$. Simply put, the first step is to form the Homotopy equation to determine $\gamma^{m}$, i.e.,

$$
\gamma^{m}-A(\eta)=p\left[\gamma^{m}-L(\gamma)-D^{\alpha}\right], p \in[0,1]
$$

where $D^{\alpha} \gamma$ is the Caputo fractional derivative of order $\alpha$, subject to the initial condition

$$
\gamma^{k}(0)=c_{k} ; k=1,2,3, \ldots, m-1 .
$$

If $m=1$ then $k=0$ and (5) can be derived to the system of equations

$$
\begin{aligned}
\gamma_{0}^{\prime}=A(\eta), \gamma_{0}(0) & =c_{0}, \\
\gamma_{1}^{\prime}=\gamma_{0}^{\prime}-\gamma_{0}-D^{\alpha} \gamma_{0}, \gamma_{1}(0) & =0 \\
\gamma_{2}^{\prime}=\gamma_{1}^{\prime}-\gamma_{1}-D^{\alpha} \gamma_{0}, \gamma_{2}(0) & =0, \text { and so forth. }
\end{aligned}
$$

Therefore, based on MHPM the solution function of (4) is given by

$$
\gamma(\eta)=\gamma_{0}(\eta)+\gamma_{1}(\eta)+\gamma_{2}(\eta)+\gamma_{3}(\eta)+\cdots
$$


Based on (6) and data in Table 1, we obtain $\alpha=0.3$, $b=22.758$ and $h=0.01$. Thus, the exponential fractional differential equation in (4) becomes

$$
\gamma^{(0.3)}+\gamma=22.758 e^{0.01 \eta}
$$

and it has a solution that converges to the function

$$
\gamma=18.189 e^{0.01 \eta}
$$

where $\gamma$ is the surface tension, $100 \eta$ is viscosity, that is if $\eta=x$ then the viscosity value is $100 x$.

\subsection{Analysis of The Solution Function}

The graph of the solution function is shown in the Figures 1 and 2 .

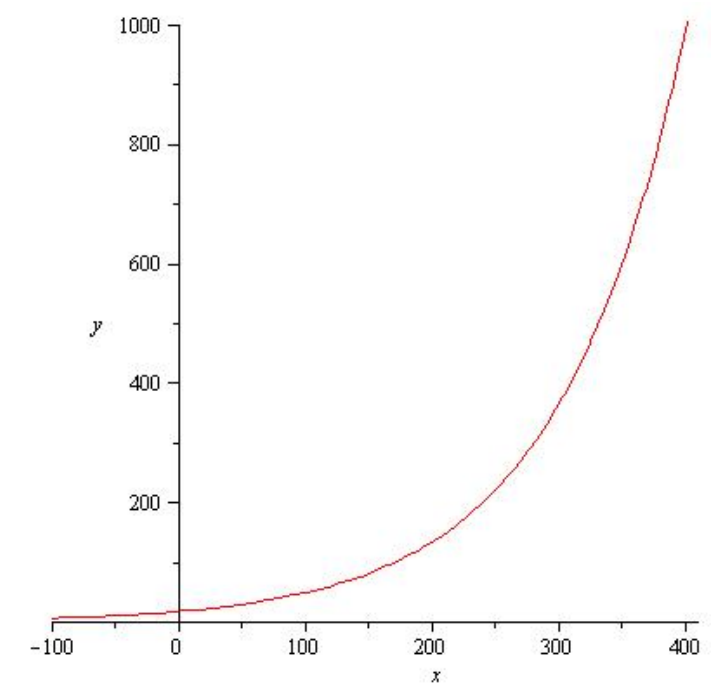

Figure 1. The actual image of the solution function.

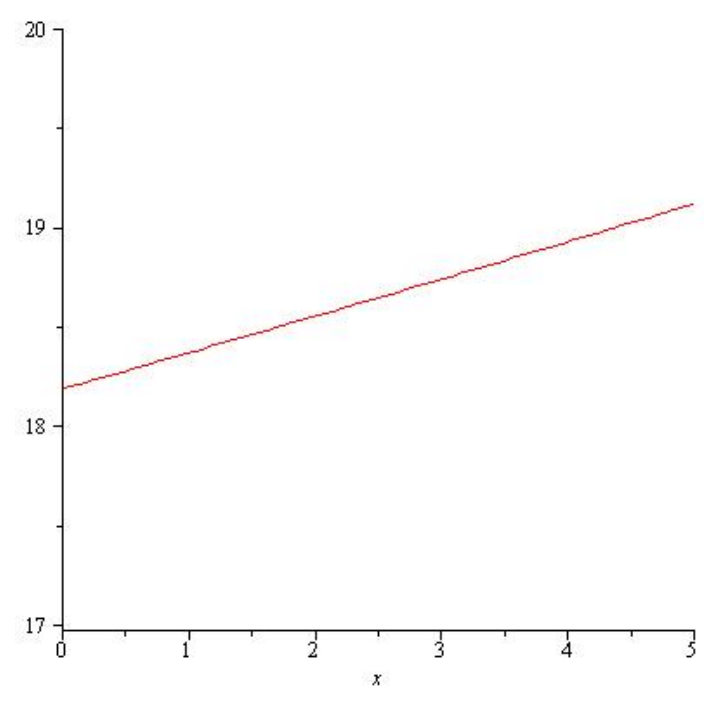

Figure 2. The image of the solution function from data sample.

Figure 1 shows the actual image of the solution function so that it looks like an exponential function. Furthermore, because the viscosity value of the sample is in the range $0<100 \eta<$ 500 , and the surface tension value is in the range $18<\gamma<20$, then the curve looks like in Figure 2.

The followings are the results of the analysis of the solution function and its graph.

1. The graph is monotonically increasing. This implies that the higher the fluid viscosity value, the greater the surface tension value.

2. The value of the fractional order is relatively small, i.e., $\alpha=0.3$, and the value of $k$ on $e^{k \eta}$ is also small, i.e., $k=0.01$. This is due to the significant ratio between surface tension and viscosity; for instance, if the viscosity value increases by 100 units, the surface tension increases by around 0.57 units.

\section{Conclusions}

Based on the previous results, there is a relationship between viscosity and surface tension of fluid, especially lubricating oil, where the relationship can be expressed in an exponential fractional differential equation model. From the graph of the solution function, we show that this relationship is directly proportional, i.e., the thicker the lubricating oil, the greater the surface tension value. The findings of this study may serve as the foundation for future research, including determining the relationship between viscosity and surface tension for other types of fluids, such as paints, and examining the effect of lubricating oil viscosity on low oil pressure.

\section{Acknowledgements}

The authors would like to thank the Rector of Universitas Padjadjaran and Director of Directorate of Research and Community Service Universitas Padjadjaran who gave funding for dissemination of this paper through Hibah Riset Data Pustaka dan Daring (RDPD) Universitas Padjadjaran with contract number: 1959/UN6.3.1/PT.00/2021.

\section{REFERENCES}

[1] Gatenby A., "How Does Surface Tension Relate to Viscosity?", CSC Scientific, 2011.

[2] Pelofsky A. H., "Surface Tension-Viscosity Relation for Liquids", Journal of Chemical \& Engineering Data, vol. 11, no. 3, pp. 394-397, 1966.

[3] Schonhorn H., "Surface Tension-Viscosity Relationship for Liquids", Journal of Chemical and Engineering Data, vol. 12, no. 4 , pp. 524-525, 1967. 
[4] Li X., Tian J., Mulero A., "Empirical correlation of the surfacetension versus the viscosity for saturated normal liquids", Fluid Phase Equilibria, vol. 352, pp. 54-63, 2013.

[5] Ghatee M. H., Bahrami M., Khanjari N., Firouzabadi H., Ahmadi Y. A., "Functionalized High-Surface-Energy Ammonium-BasedIonic Liquid: Experimental Measurement of Viscosity, Density, and Surface Tension of (2-Hydroxyethyl) ammonium Formate", Journal of Chemical \& Engineering Data, vol. 57, no. 8, pp. 2095-2101, 2012.

[6] Ghatee M. H., Zare M., Zolghadr A. R., Moosavi F., "Temperature dependence of viscosity and relation with the surfacetension of ionic liquids", Fluid Phase Equilibria, vol. 291, pp. 188-194, 2010.

[7] Hadi A., Amiri M. C., "On the relationship between surface tension and viscosity of fluids", Chemical Engineering Research Bulletin, vol. 18, no. 1, pp. 18-22, 2015.

[8] Zheng M., Tian J., Mulero Á., ”New correlations between viscosity and surface tension for saturated normal fluids", Fluid Phase Equilibria, vol. 360, pp. 298-304. 2013

[9] Queimada A. J., Marrucho I. M., Stenby E. H., Coutinho J. A. P., "Generalized relation between surface tension and viscosity: a study on pure and mixed n-alkanes", Fluid Phase Equilibria, vol. 222, pp. 161-168, 2004.

[10] Queimada A. J., "Prediction of viscosities and surface tensions of fuels using a new corresponding states model", Fuel, vol. 85, no. 5-6, pp. 874-877, 2006.

[11] Rusyaman E., Parmikanti K., Chaerani D., Supian S., "The fractional relationship between viscosity and surface tension on lubricating oils", IAENG-international Journal of Applied Mathematics, vol. 50, no. 1, 2020.

[12] Mahmood A., Parveen S., Ara A., Khan N. A., "Exact analytic solutions for the unsteady flow of a non-Newtonian fluid between two cylinders with fractional derivative model", Communications in Nonlinear Science and Numerical Simulation, vol. 14, no. 8, pp. 3309-3319, 2009.

[13] Jamil M., Khan N. A., Zafar A. A., "Translational flows of an Oldroyd-B fluid with fractional derivatives", Computers and Mathematics with Applications, vol. 62, no. 3, pp. 1540-1553, 2011.

[14] Servadei R., Valdinoci E., "Weak And Viscosity Solutions Of The Fractional Laplace Equation", Publication Matematiques, vol. 58, pp. 133-154, 2014.

[15] Mahnaz A., "Numerical Solution for solving a System of Fractional Integro-differential Equations", IAENG International Journal of Applied Mathematics, vol. 45, no. 2, pp. 85-91, 2015.
[16] Pandey P., Holm S., "Linking the fractional derivative and the Lomnitz creep law to non-Newtonian time-varying viscosity", PHYSICAL REVIEW E, vol. 94, no. 3, pp. 032606, 2016.

[17] Mainardi F., Spada G., "Creep, Relaxation and Viscosity Properties for Basic Fractional Models in Rheology", The European Physical Journal Special Topics, vol. 193, no. 1, pp. 133-160, 2011.

[18] Hayat T., Nadeem S., Asghar S., Periodic unidirectional flows af a viscoelasti fluid with the fractional Maxwell model, Applied Mathematics and Computation, vol. 151, no. 1, pp. 153-161, 2004.

[19] Khan M., Ali S. H., Fetecau C., Qi H., "Decay of potential vortex for a viscoelastic fluid with fractional Maxwell model", Applied Mathematical Modelling, vol. 33, no. 5, pp. 2526-2533, 2009.

[20] Meral F. C., Royston T. J., Magin R., 'Fractional calculus in viscoelasticity: An experimental study", Communications in Nonlinear Science and Number Simulation, vol. 15, no. 4, pp. 939-945, 2010.

[21] Craiem D., Armentano R. L., "A fractional derivative model to describe arterial viscoelasticity", Biorheology, vol. 44, no. 4, pp. 251-263, 2007.

[22] Yoon J. M., Xie S., Hrynkiv V., "A Series Solution to a Partial Integro-Differential Equation Arising in Viscoelasticity", IAENG International Journal of Applied Mathematics, vol. 43, no. 4, pp. 172-175, 2013.

[23] Wang S., Xu M., "Axial Couette flow of two kinds of fractional viscoelastic fluids in an annulus", Nonlinear Analysis: Real World Applications, vol. 10, no. 2, pp. 1087-1096, 2009.

[24] Jamil M., Khan N. A., "Slip Effects on Fractional Viscoelastic Fluids", International Journal of Differential Equations, vol. 2011, 2011.

[25] Michal S., Hana M., Jan K., "The determination of viscosity at liquid mixtures - Comparison of approaches", AIP Conference Proceedings, vol. 1189, no. 1, pp. 020035, 2017.

[26] Aleem M., Asjad M. I., Chowdhury M. S. R., Hussanan A., "Analysis of mathematical model of fractional viscous fluid through a vertical rectangular channel", Chinese Journal of Physics, vol. 61, pp. 336 - 350, 2019.

[27] Ortiz J. M., Hernández L. R., "The theorem existence and uniqueness of the solution of a fractional differential equation", Acta Universitaria, vol. 23, no. 2, pp. 16-18, 2013. 
[28] Zhang S., "Existence of a solution for the fractional differential equation with nonlinear boundary conditions", Computers and Mathematics with Applications, vol. 61, no.4, pp. 1202-1208, 2011 .

[29] Schertzer M. J., Iglesias P., ”Meta-Analysis Comparing Wettability Parameters and the Effect of Wettability on Friction Coefficient in Lubrication", Lubricants, vol. 6, no.3, pp. 70, 2018.

[30] El-Borai M. M., El-Nadi K. E., "The Parabolic Transform and Some Singular Integral Evolution Equations", Mathematics and Statistics Journal, vol. 8, no. 4, pp. 410-415, 2020. DOI: 10.13189/ms.2020.080406.

[31] Bouchenak A., Roshdi K., Mohamed A., "Fractional Fourier Series with Separation of Variables Technique and It's Application on Fractional differential Equations", WSEAS Transactions on Mathematics, vol. 20, pp. 461-469, 2021. 